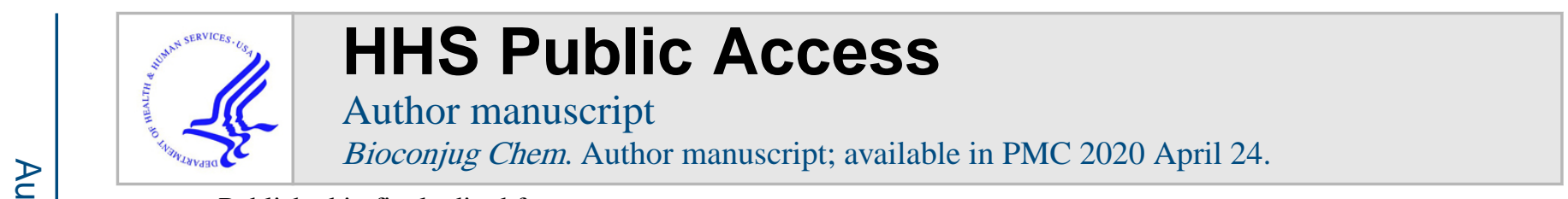

Published in final edited form as:

Bioconjug Chem. 2019 November 20; 30(11): 2822-2827. doi:10.1021/acs.bioconjchem.9b00561.

\title{
Flavin Binding Allosteric Aptamer with Noncovalent Labeling for miR Sensing
}

\author{
A. Gee ${ }^{\dagger}$, J. G. Grennell ${ }^{\dagger}$, S. Sitaula ${ }^{\dagger}$, J. Jayawickramarajah ${ }^{\ddagger}$, M. F. Ali ${ }^{\star}, \dagger$ \\ tDepartment of Chemistry, Xavier University of Louisiana, 1 Drexel Drive, New Orleans, Louisiana \\ 70125, United States \\ ‡Department of Chemistry, Tulane University, 2015 Percival Stern Hall, New Orleans, Louisiana \\ 70118, United States
}

\begin{abstract}
Modular allosteric aptamers with discrete recognition and signaling regions provide a facile method of carrying out label-free detection by forgoing complex target labeling requirements. Herein, we describe the design and function of an aptamer scaffold capable of forming a hairpin loop in the presence of FAD (the signaling trigger). The aptamer includes a recognition region for the microRNA (miR) Let-7i. Upon selective miR hybridization, the aptamer undergoes a conformational shift to release FAD and thus produce a measurable response. As a result, the described method can sensitively and selectively detect miR Let-7i with a wide linear range of 0.1 $\mathrm{pM}$ to $1 \mu \mathrm{M}$ and a detection limit of $150 \mathrm{fM}$. Additionally, this strategy was able to selectively discriminate between sequences with 1- and 2-nucleotide (nt) differences.
\end{abstract}

\section{Graphical Abstract}
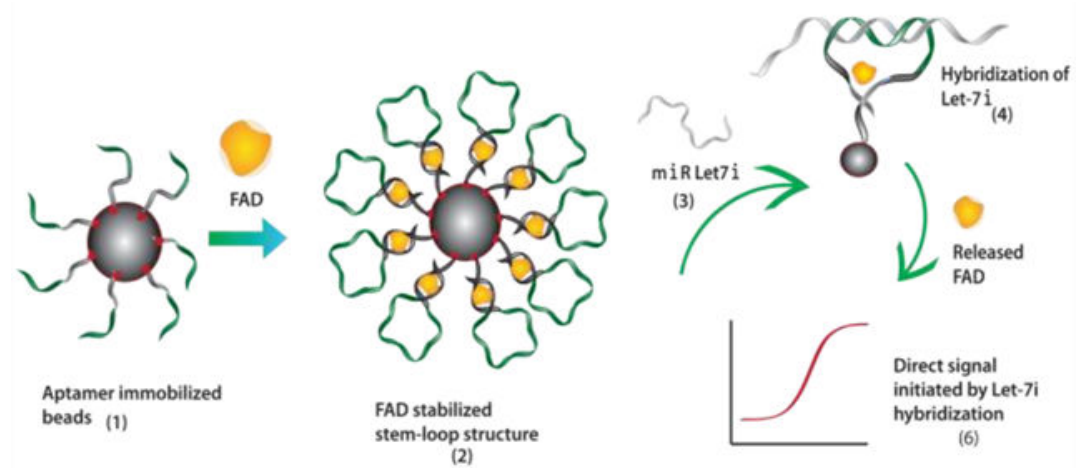

*Corresponding Author mali2@xula.edu.

Author Contributions

All authors have given approval to the final version of the manuscript.

ASSOCIATED CONTENT

Supporting Information

The Supporting Information is available free of charge on the ACS Publications website at DOI: 10.1021/acs.bioconj-chem.9b00561.

Additional ITC data on aptamer binding is provided (PDF)

The authors declare no competing financial interest. 
Early disease diagnosis has reached new horizons with the capabilities of detecting circulating miRNAs, tumor DNA, as well as specific somatic mutations for "liquid biopsies". Applications of detection systems at the nanomedicine regime require highly sensitive, quantitative, and selective detection platforms for the real-time multiplexed monitoring of target biofluids. ${ }^{1}$ There are numerous technologies that offer salient methods for detection, ${ }^{2-4}$ but often these systems are limited by complex assay conditions and are not easily adaptable to the type of target. ${ }^{5-7}$ Of the biomarkers of clinical importance, miRNAs are ubiquitous in multiple body fluids such as plasma, saliva, urine, breast milk, and cerebrospinal fluid. The robust nature of miRNAs with respect to abundance, stability in changing $\mathrm{pH}$ levels, higher resistance to enzymatic degradation, and tissue specific expression make them an ideal class of biofluid-based biomarkers. ${ }^{8,9}$

The detection of miRNAs have undergone significant evolution from the use of Northern blots to qRT-PCR methods. ${ }^{10}$ However, due to the difficulty in designing primers for short sequences, there continues to be a need for a simple sensitive system that is capable of measuring miRNA levels from biofluids in a reproducible and reliable manner. ${ }^{11}$ Some of the current strategies developed to address conventional difficulties ${ }^{12}$ have included electrochemical systems using amperometric and voltammetric methods. A variety of optical systems using fluorescence, colorimetric, and surface plasmon resonance have also been reported. In addition to these systems, other emerging technologies have included beadbased detection and amplification strategies such as rolling circle amplification (RCA) and enzymatic amplification. These amplification systems have provided a sensitive approach in response to the need for miR detection. However, the use of numerous secondary reagents and complex assay design does not allow these strategies to be easily amenable to different target sequences and requires the need for extensive optimization steps.

The necessity of detection strategies that require minimal postlabeling can be addressed with the use of modular aptamer systems, which allow for facile label-free detection by utilizing recognition and signaling domains to reflect changes in binding. Such modular systems using an aptamer toward malachite green have been pursued for the detection of small molecules such as theophylline and $\mathrm{ATP}^{13}$ as well as proteins such as hepatitis $\mathrm{C}$ helicase. ${ }^{14}$ One of the few allosteric systems that exist for miR detection, ${ }^{15}$ utilizes the streptavidin binding aptamer within a hairpin DNA switch probe that upon target sequence recognition triggers a hybridization chain reaction. More recent approaches of allosteric systems have included also using the streptavidin aptamer sequence as a fluorophore-labeled system that shows enhanced fluorescence upon phosphorylation of $\mathrm{cDNA}^{16}$ in the presence of $\mathrm{T} 4$ polynucleotide kinase. The fluorescent signal from this system requires the use of a flow cytometer which limits its flexibility.

In this work, rational-design was used to construct an allosteric aptamer for the detection of miRNA. A previously selected RNA motif ${ }^{17}$ capable of recognizing the isoalloxazine region of FAD (Figure 1A) was used as a scaffold for a new functional molecule. The detailed work by Burgstaller et al. points to the formation of an internal loop in the presence of a flavin cofactor. The binding of the flavin ligand induces a conformational change of the RNA aptamer and takes the shape of a hairpin loop structure. This binding event stabilizes the formation of a stem between U18-G19 and A25-C24 (as seen by the dotted lines in Figure 
1B). Inspired by this conformational change, we constructed an allosteric system where the previously identified RNA aptamer ${ }^{17,18}$ was redesigned to include a target-binding region (Figure 1C). Rational design allows us to predict the change in the aptamer's behavior through structural modification of joining a target-binding loop to a flavin-binding aptamer. In the presence of a flavin ligand, the modified aptamer should continue to fold into a stemloop structure albeit with an additional target binding loop. However, the hybridization of a specific target sequence to the miR binding loop should cause a structural modification and release the flavin ligand. The use of flavin as the conformational change agent is especially poignant due to our previous work where FAD can be used as a signaling trigger to modulate the activity of the enzyme glucose oxidase (GOx). ${ }^{19}$ This previous system was used within a much diffrent application using a displacement assay for ATP detection. Herein, we describe an elegant aptamer-based detection strategy for the miRNA Let-7i. The Let-7 family of miRNA's play an important role in gene regulation, cell adhesion, and muscle formation. ${ }^{20,21}$ Let-7 $\mathrm{i}$ as a target sequence has been identified as being deregulated in cancer rogression ${ }^{22-24}$ and correlated as a key marker that is modulated in traumatic brain injury patients. ${ }^{25,26}$

Our current allosteric system utilizes a stem-loop motif but avoids the necessity of fluorophore/quencher conjugations in comparison to traditional molecular beacon-based probes. ${ }^{27}$ Instead this noncovalent labeling approach uses FAD both as a conformation switch as well as a signaling trigger. The allosteric aptamers conjugated to magnetic nanoparticles provide a facile method for the detection of short miR sequences in a selective and sensitive manner.

\section{RESULTS AND DISCUSSION}

\section{Principle of Allosteric Aptamer Detection Strategy.}

The described allosteric aptamer system for the detection of miR Let-7i is schematically illustrated in Scheme 1. Aptamers (denoted as Let-7i-Apt) consisting of binding regions for both flavin and Let-7i were immobilized onto streptavidin coated magnetic beads (via a $5^{\prime}$ biotin). The addition of FAD induces a conformational change that forces a stem-loop structure to form. An important consideration here is that the modification of the aptamer to include a miR-binding region does not preclude it from binding FAD. The addition of miR Let-7i allows for hybridization of the target to the loop of the aptamer causing unraveling of the stem-loop structure. The specific hybridization of Let-7i releases FAD, which is now free to reactivate apo-GOx and produce measurable amounts of $\mathrm{H}_{2} \mathrm{O}_{2}$. In order to quantitate the amount of $\mathrm{H}_{2} \mathrm{O}_{2}$ produced, a coupled peroxidase catalyzed secondary reaction was used to reflect changes in reaction rate. The formation of the initial hairpin in the absence of a specific miR sequence is crucial toward reducing any background signal associated with an unraveled aptamer. Since only the presence of Let-7i triggers the reactivation of apo-GOx, the final changes in signal were correlated to the concentration of Let-7i present.

This simple yet elegant detection strategy is dependent on the availability of two discrete binding regions within the modified aptamer. The existing FAD binding aptamer (Figure 1B) was modified to include a Let-7i binding region with continued FAD binding ability. Thus, the modified aptamer provides allosteric binding of the Let-7i miRNA producing a 
conformational shift in the stem-binding region, which releases the prebound FAD as the signaling trigger. The modified aptamer was designed using $m$-fold. ${ }^{28}$ Continued binding of FAD to the modified Let-7i-Apt was thermodynamically characterized using isothermal titration calorimetry (ITC) (Figure S1). Calorimetric data were fit to a single set of identical sites resulting in a $K_{\mathrm{d}}$ of $\sim 60 \mu \mathrm{M}$. This $K_{\mathrm{d}}$, while weaker in comparison to the subnanomolar $(700 \mathrm{nM})$ binding of the unmodified aptamer ${ }^{18}$ to FAD, nevertheless proves efficacy for the design strategy which inserts the complementary sequence for the target of interest into the aptamer scaffold and shows continued binding of FAD.

The avidin-coated magnetic beads can be used to immobilize aptamers (via a $5^{\prime}$ biotin) in order to facilitate separation steps. In this work, this particular step serves to not only remove unbound Let-7i-Apt by using a buffer rinse but also to remove unbound FAD prior to adding the target of interest (Let-7i). The sensitivity of this binding assay toward Let-7i was determined by adding varied concentrations of the target Let-7i to the FAD-stabilized hairpin loop immobilized on the beads. Since the loop of the hairpin consists of a complementary region to the target Let-7i, the presence of Let-7i causes an initial hybridization of the miR to the loop of the hairpin. When the consequent "zipping" of the target to loop region increases, the aptamer stems are "unzipped" and the hairpin structure opens. ${ }^{29}$ The base pairing of the remaining probe and Let-7i complementary regions follows this second step. A direct effect of the hybridization of the $\mathrm{miR}$ is the release of the flavin trigger, which is able to reactivate apo-GOx as shown in Scheme 1.

\section{Analytical Performance of Allosteric Probe.}

As seen in Figure 2A, the analytical performance of the design strategy was investigated by measuring the activity of the enzyme with different amounts of Let-7i being added to the allosteric probe. As the concentration of Let-7i was increased, the enzyme activity also increased (via reactivation of apo-GOx) showing saturation in signals above concentrations of $10 \mu \mathrm{M}$ of Let-7i (Figure 2A). This trend followed closely the expectation that higher concentrations of target miR would unravel a larger number of hairpin-loop structures leading to more FAD being released and ultimately increasing the activity from the signaling enzyme.

A linear relationship between enzyme activity and concentrations of Let-7i was obtained in the range from $10^{-13} \mathrm{M}$ to $10^{-6} \mathrm{M}$ (Figure $2 \mathrm{~B}$ ) represented by $\mathrm{d} A_{\text {obs }} / \mathrm{d} t=0.1000 \lg c+1.284$ where $\mathrm{d} A_{\mathrm{obs}} / \mathrm{d} t$ is the reaction rate and $c$ is the amount of target Let-7i. The detection limit for Let-7i was calculated at $150 \mathrm{fM} \mathrm{(50} \mathrm{attomols)} \mathrm{and} \mathrm{corresponds} \mathrm{to} 3 \sigma$ above the mean background level. Thus, the displacement strategy is sensitive to the lower end of miR concentrations that are extracted from plasma and serum ( $\sim 500-700 \mathrm{fmols}$ or $\sim 3-4 \mathrm{ng} / \mu \mathrm{L})$. 8,11

\section{Specificity of Let-7i Binding Probe.}

The specificity of the proposed strategy was explored with the use of both single and double nucleotide mutations (Figure 3A). The importance of these studies are gathered from literature evidence that points to patients with early stage cancer that can present with less than one mutant template molecule per milliliters of blood plasma. ${ }^{30}$ 
To investigate the specificity, Let-7i was substituted with three other sequences consisting of single or double mutations, all with the same concentration of $0.1 \mu \mathrm{M}$. The three mismatched sequences are labeled MM1, MM2, and MM3, and the entire sequence and location of the mismatched bases is specified in the Figure 3A inset. The signal was determined from the addition of Let-7i (the perfect complement) to the Let-7i-Apt as well as sequences with either single or double base pair mutations (with differing melting temperatures). The signal from the single and double mismatch sequences were below $15 \%$ of the signal for the perfect match and demonstrated good selectivity toward the target miR.

To determine the efficacy of using the FAD displacement system with "real" samples, a spike/recovery study was carried out, by adding a known amount of Let-7i to human serum. The Let-7i was spiked at concentrations of $10^{-8} \mathrm{M}$ and $10^{-10} \mathrm{M}$, which corresponds to $10^{9}$ copies $/ \mu \mathrm{L}$ and $10^{7}$ copies $/ \mu \mathrm{L}$ respectively. Figure 3B shows that the Let-7i signal measured by the aptamer-based system corresponds to the expected amounts with $107 \%$ and $95 \%$ recovery, respectively. Additionally, calculated $95 \%$ confidence intervals indicate that the difference between the mean of the expected and measured amounts are not statistically significant. Here, it is important to note that concentrations were chosen to align with expressed levels of miRNA in clinical diseases.

Regeneration of the sensor is an important practical feature since it adds to increase throughput. The allosteric aptamer immobilized beads described were regenerated by implementing a thermal step at the end of each target recognition cycle and rehybridized with subsequent target analyte introduction (Figure 4A). This procedure was followed for 3 subsequent hybridization and rinse steps with Let-7i with the Let-7i-Apt immobilized beads. The signals from the three trials were precise with a RSD of $~ 5 \%$ (Figure 4B). However, further regeneration studies are necessary in order to test and establish the practical reusability of this approach with respect to signal drop-off and number of viable reusable cycles.

In summary, the described studies indicate that our allosteric aptamer strategy is an effective method for the detection of miRNA Let-7i. The newly designed hairpin probe has the ability to bind both the signaling molecule as well as the clinical target. The continued binding of FAD (signaling trigger) to the modified aptamer was confirmed using ITC. Let-7i binding to the Let-7i-Apt was shown to release bound FAD, which directly turned on the activity of the apo-GOx in a dose dependent manner. With the use of this noncovalent detection mechanism, a clinically relevant RNA target was detected down to $150 \mathrm{fM}$. The selectivity of the system was probed via the presence of sequences containing single and double base pair mutations. This particular design strategy presents an aptamer scaffold that can be adapted toward the detection of multiple miRs from a clinically relevant panel of markers.

\section{MATERIALS AND METHODS}

\section{General Experimental Procedure.}

MagnaBind Strepta-vidin-coated beads in phosphate-buffered saline (PBS) pH 7.5 containing $0.1 \%$ BSA, EDTA, and sodium azide were supplied by Thermo Fisher Scientific. Beads were stored at $4{ }^{\circ} \mathrm{C}$ and opened just prior to use. The binding capacity of beads is 8 
$\mathrm{nmol}$ of biotin $/ \mathrm{mL}$ of beads. The $5^{\prime}$ biotinylated, Let-7i aptamers and complementary sequences of these aptamers in desalted form were supplied by Integrated DNA Technologies (IDT) and were reconstituted in DNase, RNase, and Protease free water. Glucose oxidase from Aspergillus niger Type VI-A, flavin adenine dinucleotide disodium salt hydrate (FAD), 4-aminoantipyrine, 3,5-dichloro-2-hydroxybenzesulfonate, sodium phosphate monobasic monohydrate, and glucose were purchased from Sigma-Aldrich. Sodium phosphate dibasic heptahydrate (EMD), horseradish peroxidase (POD), and ultrapure glycerol (Invitrogen) were purchased from Thermo Fisher Scientific.

Water from Milli-Q Ultra-Pure Water Purification System was autoclaved for use in buffers and in the preparation of other reagents. Glucose assay was carried out by measuring the initial rate of the enzyme (reactivated or native) by using the kinetic mode on a Beckman Coulter DU-800 Spectrophotometer at $510 \mathrm{~nm}$. Disposable semimicro cuvettes $(1.5 \mathrm{~mL})$ of an optical path length $10 \mathrm{~mm}$ were used for absorbance measurements. The binding of aptamers to streptavidin-coated beads were measured using the NanoDrop 2000c from Thermo Fisher Scientific. MicroCal Auto-ITC 200 from GE Healthcare was used for binding studies.

\section{Glucose Assay.}

Glucose $(550 \mathrm{mM})$ was prepared in water and left to mutarotate for $3 \mathrm{~h}$. 4-Aminoantipyrine $(9.98 \mathrm{mM})$ and 3,5-dichloro-2-hydroxybenzesulfonate $(11.36 \mathrm{mM})$ stocks were prepared in water and saved at $4{ }^{\circ} \mathrm{C}$. Stock 4-aminoantipyrine $3.5 \mathrm{~mL}$ and 3,5-dichloro-2hydroxybenzene-sulfonate $3.5 \mathrm{~mL}$ were mixed together and diluted with $3 \mathrm{~mL}$ phosphate buffer (0.1M, pH 6.2) just prior to the assay. POD was diluted to $60 \mathrm{U} / \mathrm{mL}$.

A typical glucose assay was carried out by mixing the following reagents to a total volume of $1 \mathrm{~mL}$ in a $1.5 \mathrm{~mL}$ disposable cuvette. Diluted and premixed solutions of 4aminoantipyrine and 3,5-dichloro-2-hydroxybenzensulfonate in $0.1 \mathrm{M}$ phosphate buffer $\mathrm{pH}$ $6.2(500 \mu \mathrm{L})$ was transferred to a cuvette. Solutions of glucose $(550 \mathrm{mM}, 50 \mu \mathrm{L})$ and POD (60U/mL, $50 \mu \mathrm{L}$ ) were added to the cuvette followed by the addition of water $(390 \mu \mathrm{L})$. Reactivated apo-GOx (10 $\mu \mathrm{L})$ was added to cuvette and initial rate of change in absorbance was measured for $2 \mathrm{~min}$.

\section{FAD Displacement Experiment.}

The experimental details of dose-dependent FAD displacement by Let-7i binding are described herein. MagnaBind beads ( $250 \mu \mathrm{L}, 2 \mathrm{nmol}$ biotin) were transferred to five different tubes labeled A Let-7i, B Let-7i, C Let-7i, control with aptamer, and control without aptamer, respectively. The beads were washed with $0.1 \mathrm{M}$ phosphate buffer $\mathrm{pH} 7.4$ $\left(250 \mu \mathrm{L} \times 2\right.$ ) followed by $250 \mu \mathrm{L}$ binding buffer (Tris $\mathrm{HCl}, 0.5 \mathrm{M} ; \mathrm{NaCl}, 1 \mathrm{M} ; \mathrm{MgCl}_{2}, 0.1$ $\mathrm{M} ; \mathrm{pH}$ 7.50). After the washing step, $5^{\prime}$-biotinylated Let-7i aptamer $(135 \mu \mathrm{L}, 17 \mu \mathrm{M})$ was added to the beads contained within the three reaction tubes. The two control tubes contained only $135 \mu \mathrm{L}$ of binding buffer. The reaction and control tubes were incubated at $50{ }^{\circ} \mathrm{C}$ for $30 \mathrm{~min}$ followed by $2 \mathrm{~h}$ at room temperature. Excess $5^{\prime}$-biotinylated Let-7i aptamer was separated from the beads by using a magnet to separate the bead and solution slurry. Beads in the reaction tubes were washed with binding buffer $(250 \mu \mathrm{L})$. The remaining 
buffer was completely removed from the beads using the magnet. Solutions of FAD (150 $\mu \mathrm{L}$, $1.2 \times 10^{-5} \mathrm{M}$ ) were added to each tube containing Let-7i aptamer conjugated to MagnaBind. The excess unbound FAD supernatant (FS) was removed from the beads using the magnet and saved in reaction tubes labeled as FS-A, FS-B, FS-C, FS-control with aptamer and FScontrol without an aptamer. A glucose assay was performed using the FS samples. The beads were washed with binding buffer $(250 \mu \mathrm{L} \times 2)$. Solutions of Let-7i $(50 \mu \mathrm{L})$ at specified concentrations were added in replicate to the tubes containing the Let-7i aptamer/FAD bead complex and control tubes. The control tubes only received the binding buffer solution (50 $\mu \mathrm{L})$. The respective reaction supernatants were removed from the beads and collected in separate tubes labeled appropriately for each of the replicates. Multiple concentrations are analyzed in additional replicate tubes. Solutions $(20 \mu \mathrm{L})$ of the supernatant containing displaced FAD were transferred to $0.5 \mathrm{~mL}$ reaction tubes, respectively. Aliquots of apo-GOx ( $20 \mu \mathrm{L}, 2 \mathrm{mg} / \mathrm{mL}$ ) were added to each individual tube containing the displaced (and control) FAD solutions. All the tubes were incubated at room temperature for $2 \mathrm{~h}$. Simultaneously, solutions of FAD $1.2 \times 10^{-5} \mathrm{M}$ in binding buffer (positive control) and an aliquot of only binding buffer (negative control) were also incubated with apo-GOx $(2 \mathrm{mg} / \mathrm{mL})$. The previously described glucose assay was carried out with $10 \mu \mathrm{L}$ of the reactivated apo-GOx using freshly prepared reagents. Total reaction times are approximately $8 \mathrm{~h}$.

\section{Selectivity Studies.}

For the selectivity experiments mutated Let-7i sequences were used to displace FAD in addition to Let-7i. The remaining procedures were similar to those described in the previous section. Let-7i was mutated with single and double pair mismatches at different positions. In this particular experiment, mismatch sequences $(50 \mu \mathrm{L})$ at a concentration of $0.1 \mu \mathrm{M}$ were added to the experimental reaction tubes. FAD displaced by mismatch sequences were used for reactivation of apo-GOx as previously described.

\section{Recovery Assay.}

For the recovery experiments, Let-7i $(0.01 \mu \mathrm{M}$ and $0.0001 \mu \mathrm{M})$ in 1:10 AB human serum:binding buffer were used to displace FAD. The remaining procedures (including sample volumes) were similar to those described in the FAD Displacement Experiment section.

\section{Isothermal Titration Calorimetry (ITC).}

Nonbiotinylated Let-7i aptamer solutions $(400 \mu \mathrm{L}, 50 \mu \mathrm{M})$ were prepared in binding buffer (Tris $\left.\mathrm{HCl}, 0.5 \mathrm{M} ; \mathrm{NaCl}, 1 \mathrm{M} ; \mathrm{MgCl}_{2}, 0.1 \mathrm{M} ; \mathrm{pH} 7.50\right)$. Let-7i aptamer $200 \mu \mathrm{L}(700 \mu \mathrm{M})$ and FAD $200 \mu \mathrm{L}(900 \mu \mathrm{M})$ solutions were also prepared in the binding buffer. All the samples and buffers for the washing syringe were sonicated for $15 \mathrm{~min}$ at RTP. A solution of Let-7i (400 $\mu \mathrm{L}$ ) was loaded in a deep well plate assigned as the target, while FAD solutions were loaded in a deep well plate assigned as the ligand, for syringe injection. The first injection was set at $0.1 \mu \mathrm{L}$, and the remaining 18 injections were set at $2 \mu \mathrm{L}$ for total injections of 19 . The FAD was injected to the cell containing the Let-7i aptamer solution at $25^{\circ} \mathrm{C}$. Upon completion of the injections, the data was processed using MicroCal Auto-iTC Origin Software. 


\section{Supplementary Material}

Refer to Web version on PubMed Central for supplementary material.

\section{ACKNOWLEDGMENTS}

M.F.A. would like to acknowledge the NIH BUILD Pilot Program UL1GM118967 and NIH 1SC2GM103704-03. The research was supported in part via funds from NASA-MIRO program NNX15AP44A-000006. A.G. and J.G.G. were supported with funds from NIH BUILD program 5RL5GM118966. A.G. was also supported via NIH BUILD TL4GM118968. We would like to acknowledge Dr. Robert Blake II in the College of Pharmacy at Xavier for generous use of the ITC and Nancy Nguyen and Jessica Anderson for helpful discussions.

\section{REFERENCES}

(1). Martinez A, Phillips S, Whitesides GM, and Carrilho E. (2010) Diagnostics for the developing world: microfludic paper-based analytical devices. Anal. Chem 82, 3-10. [PubMed: 20000334]

(2). Leng Y, Sun K, Chen X, and Li W. (2015) Suspension arrays based on nanoparticle-encoded microspheres for high-throughput multiplexed detection. Chem. Soc. Rev 44 (15), 5552-95. [PubMed: 26021602]

(3). Freeman R, Sharon E, Tel-Vered R, and Willner I. (2009) Supramolecular cocaine-aptamer complexes activate biocatalytic cascades. J. Am. Chem. Soc 131 (14), 5028-5029. [PubMed: 19309141]

(4). Bogomolova A, and Aldissi M. (2011) Real-time aptamer quantum dot fluorescent flow sensor. Biosens. Bioelectron 26 (10), 4099-4103. [PubMed: 21515039]

(5). Hunt EA, Broyles D, Head T, and Deo SK (2015) MicroRNA Detection: Current Technology and Research Strategies. Annu. Rev. Anal. Chem 8, 217-37.

(6). Li B, Ellington AD, and Chen X. (2011) Rational, modular adaptation of enzyme-free DNA circuits to multiple detection methods. Nucleic Acids Res. 39 (16), e110. [PubMed: 21693555]

(7). Jokerst JV, Cauwenberghs N, Kuznetsova T, Haddad F, Sweeney T, Hou J, Rosenberg-Hasson Y, Zhao E, Schutt R, Bolli R, Traverse JH, Pepine CJ, Henry TD, Schulman IH, Moye L, Taylor DA, and Yang PC (2017) Circulating Biomarkers to Identify Responders in Cardiac Cell Therapy. Sci. Rep 7, 1 DOI: 10.1038/s41598-017-04801-7. [PubMed: 28127051]

(8). Weber JA, Baxter DH, Zhang S, Huang DY, How Huang K, Jen Lee M, Galas DJ, and Wang K. (2010) The microRNA spectrum in 12 body fluids. Clin. Chem 56 (11), 1733-41. [PubMed: 20847327]

(9). Liu WJ, Xu Q, Sun LP, Dong QG, He CY, and Yuan Y. (2015) Expression of serum let-7c, let-7i, and let-7f microRNA with its target gene, pepsinogen $\mathrm{C}$, in gastric cancer and precancerous disease. Tumor Biol. 36 (5), 3337-43.

(10). Pritchard CC, Cheng HH, and Tewari M. (2012) MicroRNA profiling: approaches and considerations. Nat. Rev. Genet 13 (5), 358-69. [PubMed: 22510765]

(11). Kilic T, Erdem A, Ozsoz M, and Carrara S. (2018) microRNA biosensors: Opportunities and challenges among conventional and commercially available techniques. Biosens. Bioelectron 99, 525-546. [PubMed: 28823978]

(12). Jokerst JV, Chen Z, Xu L, Nolley R, Chang E, Mitchell B, Brooks JD, and Gambhir SS (2015) A magnetic bead-based sensor for the quantification of multiple prostate cancer biomarkers. PLoS One 10 (9), e0139484.

(13). Stojanovic MN, and Kolpashchikov DM (2004) Modular aptameric sensors. J. Am. Chem. Soc 126, 9266-9270. [PubMed: 15281816]

(14). Sook Bang G, Cho S, Lee N, Lee B-R, Kim J-H, and Kim B-G (2013) Rational design of modular allosteric aptamer sensor for label-free protein detection. Biosens. Bioelectron 39 (1), 44-50. [PubMed: 22819625]

(15). Cai S, Cao Z, Lau C, and Lu J. (2014) Label-free technology for the amplified detection of microRNA based on the allosteric hairpin DNA switch and hybridization chain reaction. Analyst 139 (22), 6022-7. [PubMed: 25270599] 
(16). Gao M, Guo J, Song Y, Zhu Z, and Yang CJ (2017) Detection of T4 Polynucleotide Kinase via Allosteric Aptamer Probe Platform. ACS Appl. Mater. Interfaces 9 (44), 38356-38363.

(17). Burgstaller P, and Famulok M. (1996) Structural characterization of a flavin-specific RNA aptamer by chemical probing. Bioorg. Med. Chem. Lett 6 (10), 1157-1162.

(18). Anderson PC, and Mecozzi S. (2005) Identification of a 14mer RNA that recognizes and binds flavin mononucleotide with high affinity. Nucleic Acids Res. 33 (22), 6992-6999. [PubMed: 16377778]

(19). Sitaula S, Branch SD, and Ali MF (2012) GOx signaling triggered by aptamer-based ATP detection. Chem. Commun 48, 9284-9286.

(20). Thammaiah CK, and Jayaram S. (2016) Role of let-7 family microRNA in breast cancer. Noncoding RNA Research 1, 77-82. [PubMed: 30159414]

(21). Boyerinas B, Park SM, Hau A, Murmann AE, and Peter ME (2010) The role of let-7 in cell differentiation and cancer. Endocr.-Relat. Cancer 17 (1), F19-36. [PubMed: 19779035]

(22). Mahn R, Heukamp LC, Rogenhofer S, von Ruecker A, Muller SC, and Ellinger J. (2011) Circulating microRNAs in Serum of Patients with Prostate Cancer. Urology 77 (5), e9-e16.

(23). Volinia S, Calin GA, Liu C-G, Ambs S, Cimmino A, Petrocca F, Visone R, Iorio M, Roldo C, Ferracin M, Prueitt RL, Yanaihara N, Lanza G, Scarpa A, Vecchione A, Negrini M, Harris CC, and Croce CM (2006) A microRNA expression signature of human solid tumors defines cancer gene targets. Proc. Natl. Acad. Sci. U. S. A 103, 2257-2261. [PubMed: 16461460]

(24). Baltimore D, Boldin MP, O’Connell RM, Rao DS, and Taganov KD (2008) MicroRNAs: new regulators of immune cell development and function. Nat. Immunol 9 (8), 839-845. [PubMed: 18645592]

(25). Balakathiresan N, Bhomia M, Chandran R, Chavko M, McCarron RM, and Maheshwari RK (2012) MicroRNA Let-7i is a promising serum biomarker for blast-induced traumatic brain injury. Journal of Neurotrauma 29, 1379-1387. [PubMed: 22352906]

(26). Redell JB, Moore AN, Ward NH, Hergenroeder GW, and Dash PK (2010) Human Traumatic Brain Injury alters plasma microRNA levels. Journal of Neurotrauma 27, 2147-2156. [PubMed: 20883153]

(27). Wang K, Tang Z, Yang CJ, Kim Y, Fang X, Li W, Wu Y, Medley CD, Cao Z, Li J, Colon P, Lin H, and Tan W. (2009) Molecular Engineering of DNA: Molecular Beacons. Angew. Chem., Int. Ed 48, 856-870.

(28). Zuker M. (2003) Mfold web server for nucleic acid folding and hybridization prediction. Nucleic Acids Res. 31 (13), 3406-3415. [PubMed: 12824337]

(29). Tsourkas A, Behlke MA, and Bao G. (2002) Structure-function relationships of shared-stem and conventional molecular beacons. Nucleic Acids Res. 30 (19), 4208-4215. [PubMed: 12364599]

(30). Cohen JD, Javed AA, Thoburn C, Wong F, Tie J, Gibbs P, Schmidt CM, Yip-Schneider MT, Allen PJ, Schattner M, Brand RE, Singhi AD, Petersen GM, Hong SM, Kim SC, Falconi M, Doglioni C, Weiss MJ, Ahuja N, He J, Makary MA, Maitra A, Hanash SM, Dal Molin M, Wang Y, Li L, Ptak J, Dobbyn L, Schaefer J, Silliman N, Popoli M, Goggins MG, Hruban RH, Wolfgang CL, Klein AP, Tomasetti C, Papadopoulos N, Kinzler KW, Vogelstein B, and Lennon AM (2017) Combined circulating tumor DNA and protein biomarker-based liquid biopsy for the earlier detection of pancreatic cancers. Proc. Natl. Acad. Sci. U. S. A 114 (38), 10202-10207. 


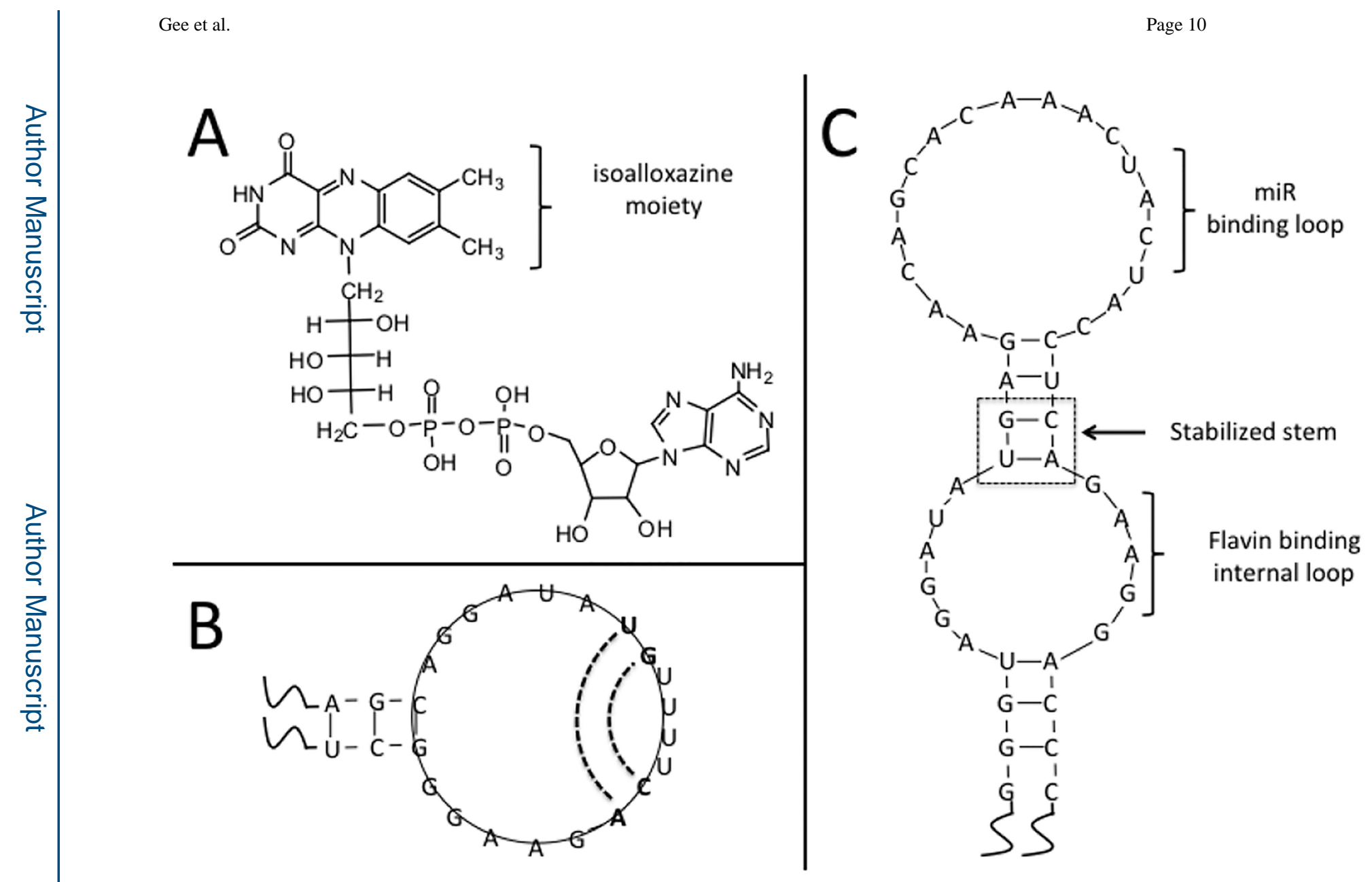

Figure 1.

(A) Structure of flavin adenine dinucleotide, and (B) scheme of original flavin binding aptamer. The dotted lines show the bases that are paired upon flavin binding. (C) Allosteric aptamer modified via rational design by joining a miR binding loop to an aptamer with a flavin binding region. This aptamer uses the bases from the original aptamer to form a stable stem. 


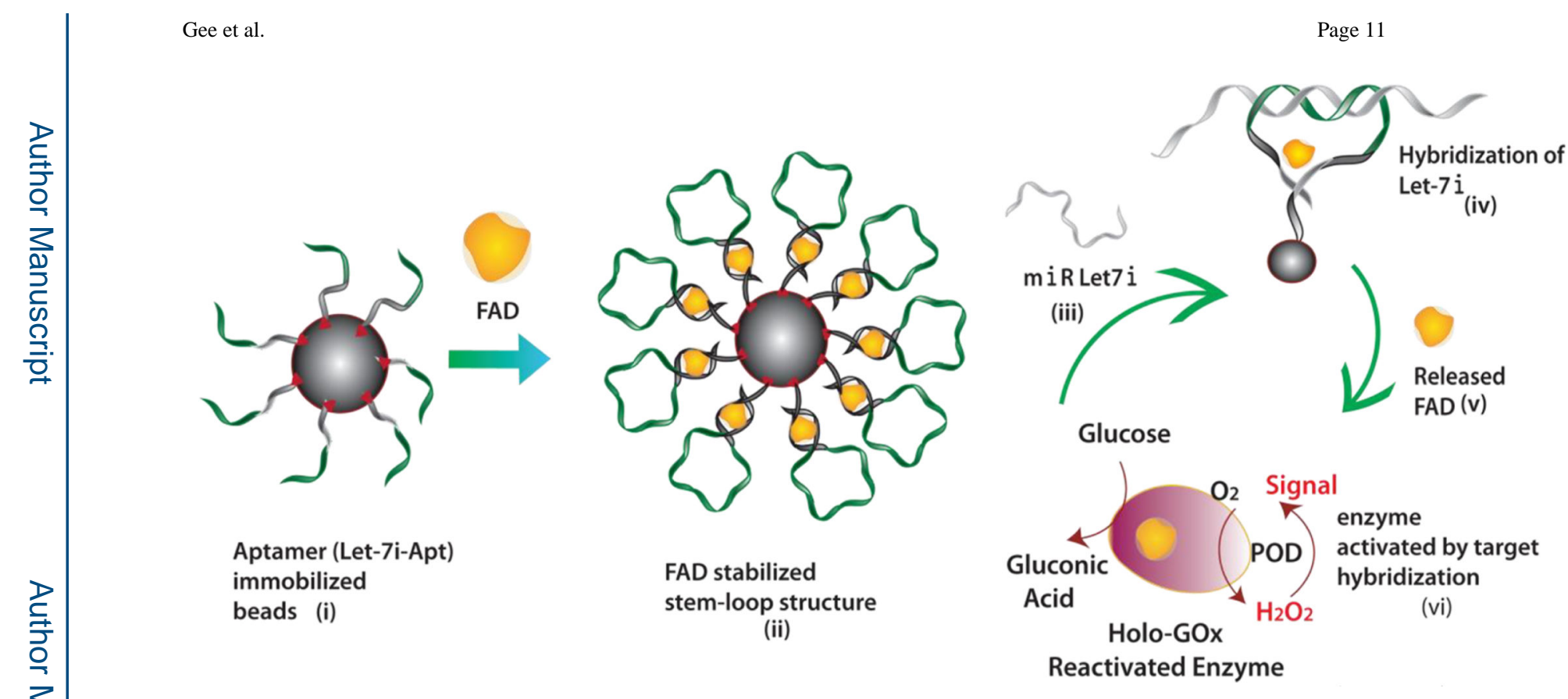

Scheme 1. Design of Allosteric Aptamer Systems Using Rational Design for the Detection of miR's ${ }^{\mathrm{a}}$

${ }^{a}$ Allosteric aptamers (Let-7i-Apt) conjugated to nanobeads (i) were stabilized into stem-loop structures via addition of FAD (ii). The presence of target sequence (iii) allows for hybridization to the loop of the aptamer thus unraveling the stem (iv) and relasing FAD (v). The free FAD is able to reactivate inactive apo-GOx and produce a measurable signal. 

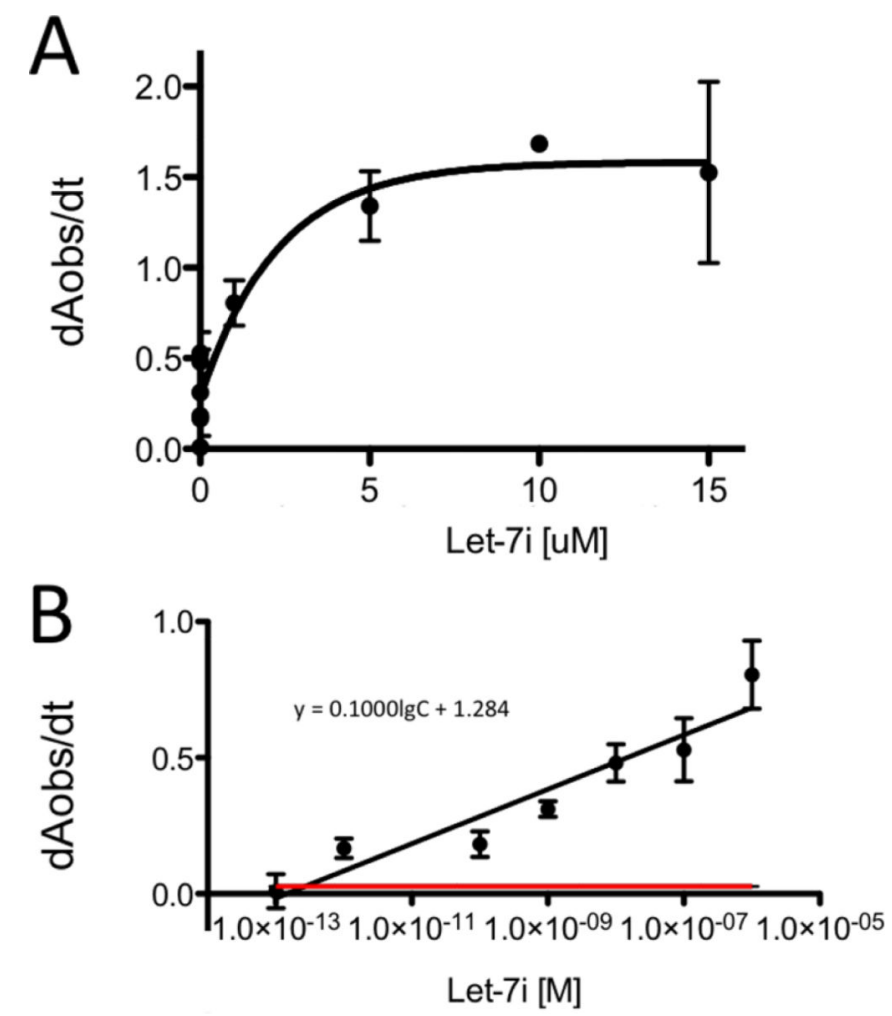

Figure 2.

(A) Dose-dependent curve of initial velocity versus Let-7i concentration. Error bars were obtained from three or more trials. (B) Response over the $10^{-13}-10^{-6} \mathrm{M}$ linear range for Let-7i. The red line indicates the LOD (3 SD above the blank signal). 

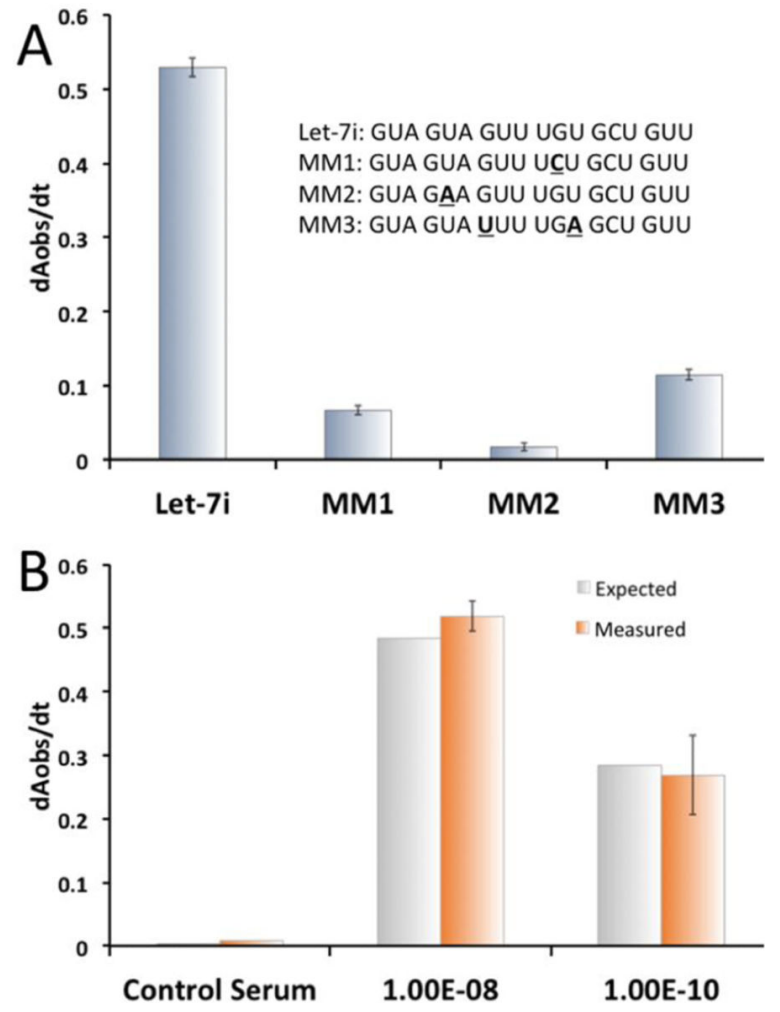

Figure 3.

(A) Bar graphs showing specificity of detection strategy in the presence of $0.1 \mu \mathrm{M}$ Let-7i and mismatched sequences MM1, 2, and 3. The mismatched nucleotides are in bold and underlined and $(\mathrm{B})$ recovery results of the detection strategy with Let $7 \mathrm{i}$ concentrations $10^{-8}$ and $10^{-10} \mathrm{M}$. 

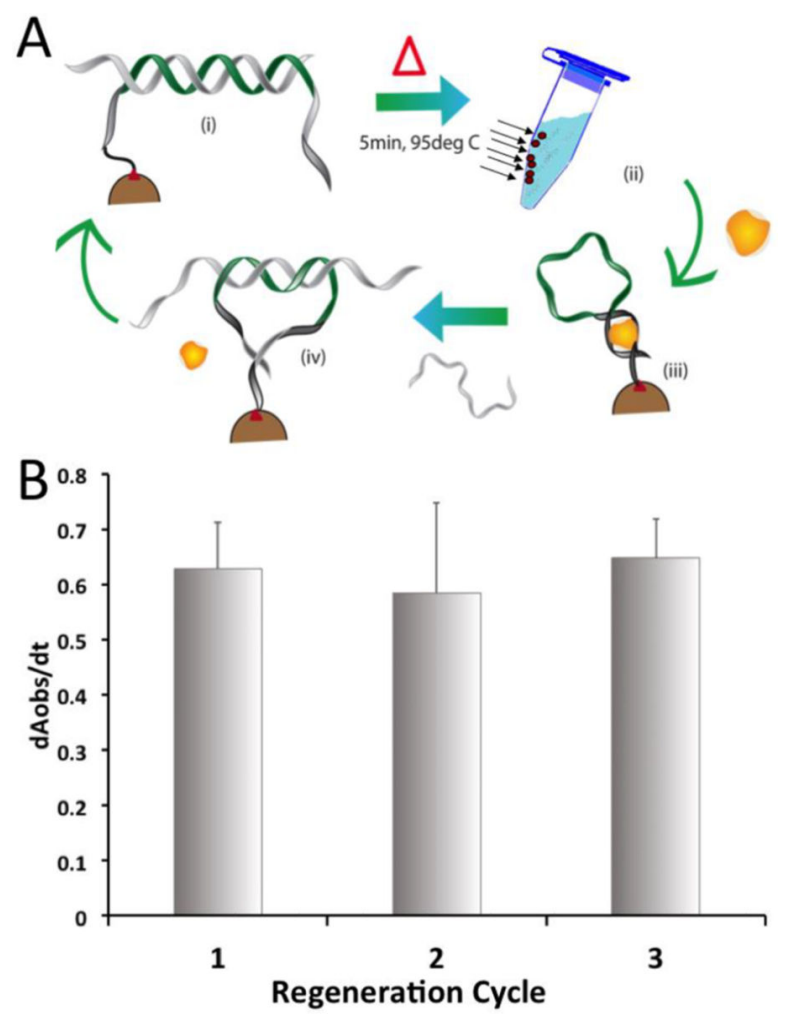

Figure 4.

(A) Regeneration cycle initiated via (i) unraveling the target/aptamer complex using a 5 min heating step; (ii) separating the released miR and any residual FAD by using a magnet to separate the beads; (iii) equilibrating the aptamer sequences with FAD which are ready for introduction of miR; and (iv) presence of specific target miR causes a structural shift and releases FAD which can be detected as previously mentioned. (B) Regeneration cycle repeated three times. Bar graphs show the signal produced with each repeated regeneration cycle. 